\title{
A case of opsoclonus myoclonus syndrome
}

\author{
A N K De Silva ${ }^{1}$, R N Morawakkorala ${ }^{2}$, K A N S Kannangara ${ }^{2}$, K Guruge $^{3}$ \\ Sri Lanka Journal of Child Health, 2008; 37: 57-8
}

(Key words: opsoclonus myoclonus syndrome)

\section{Introduction}

'Opsoclonus myoclonus syndrome' (OMS), also known as 'myoclonic encephalopathy of Kinsbourne', and 'dancing eyes-dancing feet syndrome', is a very rare disorder commonly associated with paraneoplastic features of neuroblastoma. It has a devastating and debilitating onset but is generally non fatal. Symptoms are steroid responsive and recovery from acute symptoms is good. Children suffer lifelong neurological sequelae.

\section{Case report}

A previously healthy 16 month old baby girl with normal developmental milestones, presented to a local hospital with a febrile illness, followed by unsteady gait for 3 days. It was managed as viral cerebellitis with IV aciclovir and dexamethasone. CT scan of brain done at that time was normal. She had shown a gradual recovery and was sent home within 10 days.

She had remained well for nearly one month when she was readmitted with unsteady gait, dancing eye movements and titubation. After admission, she had developed three generalised tonic clonic seizures and was transferred to the Lady Ridgeway Hospital. CT and MRI scans of the brain were normal. At this juncture parents decided to seek ayurvedic treatment and left against medical advice.

After 5 weeks, she was readmitted with fever and severe vomiting. She was very irritable and restless, had difficulty falling asleep, was extremely ataxic and could not be held in one position even with support. The eye movements were chaotic and she had myoclonic jerks. Mother was very concerned that the child had drooling and had become mute. There were no other focal neurological signs. There was no hepatosplenomegaly, lymphadenopathy or other abdominal masses. The possibility of neuroblastoma was excluded by the normal blood picture, bone marrow, urinary catecholamines and ultrasound scan of abdomen. A provisional diagnosis of OMS was made and an occult neuroblastoma was ruled out after performing CT scan of chest and abdomen. Screening for Japanese encephalitis, cytomegalovirus and Epstein Barr virus (EBV) turned out negative.

${ }^{1}$ Senior Registrar, ${ }^{2}$ Registrars, ${ }^{3}$ Consultant Paediatrician, Lady Ridgeway Hospital for Children, Colombo.

(Received on 11 April, 2007. Accepted on 20 May 2007)
She was started on ACTH subcutaneous injections, $75 \mathrm{mg}$ twice daily, continued for 1 week. The same dose was continued once daily for a further week and was then reduced to every other day injections. Within 10 days of treatment there was a significant response. Opsoclonus gradually disappeared and child became able to fix and follow objects. She started to walk with support at the end of 2 weeks and could stand on her own while on every other day regimen. During the $5^{\text {th }}$ week of treatment, when the dose was being further tailed off, she could walk on her own.

\section{Discussion:}

The average age of onset of OMS is 19 months with a range of 6-36.months. The pathophysiology of OMS has been described by autoantibodies ${ }^{2}$ directed against neural antigens $^{3}$ in cerebellar Purkinje cells, cerebral cortical neurons and axons. The immune mechanism may be a type 11 or IV hypersensitivity. It is hypothesized that a viral infection (EBV, Coxsackie B, enterovirus) or a tumour initiates the cascade of immune reactions.

Tumours are found in about half of the cases of opsoclonus myoclonus. The most common tumours in children with OMS are neuroblastoma and ganglioneuroblastoma, which often occur in the chest but also in abdomen and pelvis. The body has greater success in eradicating a neuroblastoma than any other tumour indicating a strong immune response. Overall incidence of OMS in children with neuroblastoma is approximately 3\%. When neuroblastoma is associated with OMS, the prognosis is favourable with $90 \%$ showing long term survival. But $70 \%$ of these children suffer lifelong neurological sequelae with impaired motor, cognitive, language and behavioural problems. In $50 \%$ of OMS a tumour is not detectable but the possibility remains of a low grade tumour which has regressed spontaneously before detection.

Currently there is no well accepted treatment in OMS. Generally treatment consists of immunosupression ${ }^{4}$ to reduce the formation of antibodies and promote its removal. ACTH and corticosteroids lead to rapid improvement of neurological symptoms but most patients have frequent relapses following tapering or withdrawal of drugs. ACTH acts as a direct immunosuppressive and inhibits the antibody response to T-cell dependant antigens. Corticosteroids decrease the lymphocyte differentiation and proliferation, inhibit phagocytosis and suppress production of interleukins. To overcome the problem of relapses ACTH is given in high doses over a longer period, gradually tailing off over 1 year. 
Treatment with intravenous immunoglobulin, plasmapheresis ${ }^{5}$ immunoadsorption and oral immunosuppressive agents have been used to treat resistant cases. Medications to avoid are midozolam and melatonin.

\section{References}

1. Kinsbourne M. Myoclonic encephalopathy of infants. J Neurol Neurosurg Psychiatry 1962; 25: 271-6.

2. Bataller L, Rosenfeld M R, Graus F, Vilchez J J, Cheung V, Dalmau J. Autoantigen diversity in the opsoclonus myoclonus svndrome. Ann Neurol 2003; 53: $347-53$.
3. Connolly A M, Pestronk A, Mehta S, Pranzatelli M R, Noetzel M J. Serum autoantibodies in childhood opsoclonus- myoclonus syndrome: an analysis of antigenic targets in neural tissues. J Pediatr 1997; 130: $878-84$.

4. Pranzatelli M R. The immunopharmacology of the opsoclonus-myoclonus syndrome. Clin Neuropharmacol 1996; 19: 1-47.

5. Yiu V W Y, Kovithavongs $\mathrm{T}$, McGonigle $\mathrm{L} \mathrm{F}$, Ferriera P. Plasmapheresis as an effective treatment for idiopathic opsoclonus-myoclonus syndrome. Pediatr Neurol 2001; 24:72-4. 